\title{
LA FORMACIÓN DE PROFESORES DE MATEMÁTICA DESDE EL ÁMBITO DE LA UNIVERSIDAD COMUNITARIA INTERCULTURAL
}

William Oswaldo Flores López ${ }^{1}$

\section{Resumen}

En este artículo se analizó la relación entre la formación de profesores de matemática con las funciones sustantivas de la Universidad Comunitaria Intercultural. Se trata de un estudio cualitativo con un enfoque hermenéutico donde se realizó un análisis de contenido de libros, artículos científicos, artículos de prensa, leyes, currículo y programas de formación de profesores, políticas y normas institucionales. Los resultados certifican que la educación y la formación de profesores de matemáticas desde el ámbito de la Universidad Comunitaria Intercultural, se convierte en un sistema de práctica compartida entre la universidad, la comunidad, el Estado, la sociedad civil organizada y el sector productivo y empresarial. Todo este planteamiento nos permite avalar la necesidad de formar profesores de matemáticas desde la perspectiva de comunidades de aprendizaje; creación y recreación de conocimientos, saberes y prácticas; estrategia de acompañamiento e incidencia social y comunitaria; gestión y comunicación intercultural para el desarrollo institucional; cooperación, solidaridad y complementariedad nacional e internacional.

Palabras claves: Comunidades de aprendizaje; creación y recreación de conocimientos, saberes y prácticas; estrategia de acompañamiento e incidencia social y comunitaria; gestión y comunicación intercultural; cooperación, solidaridad y complementariedad.

\section{Summary}

In this article the relationship between the formation of mathematics teachers with the substantive functions of the Intercultural Community University was analyzed. This is a qualitative study with a hermeneutic approach where a content analysis of books, scientific articles, press articles, laws, curriculum and teacher training programs, policies and institutional standards was performed. The results certify that the education and training of mathematic teachers from the academic ambit of the Intercultural Community University becomes a shared system practice between the university, the community, the State, organize civil society, the productive and business

Máster en Investigación en Didácticas de las Ciencias Experimentales y Matemática, Gestor de Proyectos de la Dirección de Cooperación Externa de la URACCAN, william.flores@uraccan.edu.ni 
sector. Throughout this approach enables us to endorse the need to train mathematic teachers from the perspective of learning communities, creation and recreation of knowledge, ancestral knowledge a skills, social and communitarian accompaniment and advocacy strategy, management and intercultural communication for institutional development, cooperation, national and international solidarity and complementarity.

Keywords: Learning communities, creation and recreation of knowledge, ancestral knowledge a skills, social and communitarian accompaniment and advocacy strategy, management and intercultural communication for institutional development, cooperation, national and international solidarity and complementarity.

\section{Introducción}

En la presente investigación se hace una reflexión acerca de los procesos de formación de profesores de Matemática desde el ámbito de la Universidad Comunitaria Intercultural. El camino que se señala puede ser asumido como una posibilidad de análisis y reflexión sobre la importancia de reconocer, integrar y articular las funciones sustantivas de la URACCAN en la formación de profesores de Matemática. Para ello, se hace referencia a la formación desde un punto de vista de comunidades de aprendizaje; creación y recreación de conocimientos, saberes y prácticas; estrategia de acompañamiento e incidencia social y comunitaria; gestión y comunicación intercultural para el desarrollo institucional; cooperación, solidaridad y complementariedad nacional e internacional.

\section{Revisión de literatura}

Históricamente los pueblos indígenas, afrodescendientes, mestizos y comunidades étnicas que habitan la Costa Caribe de Nicaragua han enfrentado enormes limitaciones para ejercer el derecho a la educación. En los años 70 un grupo de ciudadanos de la Costa Caribe Nicaragüense impulsaron una campaña que culminó con la extensión de la Universidad Nacional Autónoma de Nicaragua (UNAN) hacia la Región, ofertando cursos para la formación de profesores. Posteriormente se llevaron a cabo otros intentos de la UNAN en los años 80 con el Programa Universitario de Educación a Distancia (PRUEDIS). En 1992 se implementó el Centro Universitario de la Región Autónoma de la Costa Caribe Norte (CUR-RACCN). Los estudiantes en estas iniciativas tuvieron muchos obstáculos que no permitieron que culminaran sus estudios a pesar de múltiples esfuerzos (URACCAN, 2004).

En 1987 se promulgó en Nicaragua una nueva Constitución Política en un contexto de revolución sandinista, para la conducción más democrática del país, plasmándose principios, garantías que aseguraría la plena vigencia de los derechos humanos individuales y colectivos de los pueblos indígenas, afrodescendientes y comunidades étnicas de la Costa Caribe Nicaragüense, elevando el respeto a la diversidad étnica 
y cultural del país a través del establecimiento, por la Asamblea Nacional, de un Régimen de Autonomía en ámbito de acción de 50\% del territorio nacional, para la libre autodeterminación de los habitantes de estos territorios en la salud, en la educación, en la cultura, en la producción, en lo ecológico y en lo administrativo de justicia (Gaceta, 2007).

En Sánchez (2009) se hace énfasis en que Nicaragua es el primer país de América Latina y el Caribe en el que se establece un régimen de autonomía, lo que implica una diversidad de planteamientos, atribuciones y facultades administrativas ejercidas por los órganos de gobiernos de la Costa Caribe. Significa que la autonomía no debe entenderse como un acto único, sino como producto de un proceso en curso en que el traspaso de las facultades administrativas, y de los servicios correspondientes del estado central a los gobiernos regionales aún requiere avances.

En este contexto la educación ha venido siendo una de las demandas centrales en el proceso de definición de los derechos de la Autonomía Multiétnica, y las propuestas planteadas abarcan desde la reforma curricular, el respeto a las formas de pedagogía endógenas, el uso de los idiomas indígenas y creoles, la formación y capacitación del personal docente, hasta la elaboración de materiales didácticos culturalmente aceptables. Así mismo se propone que la educación en todos los niveles, responda a la preservación, fomento y desarrollo de las culturas ancestrales y sea diseñada, implementada y ejecutada por los mismos pueblos, para contar así con recursos humanos autóctonos, capaces de definir y ejecutar el modelo de desarrollo acorde a las condiciones de la Costa Caribe Nicaragüense. La autonomía regional hizo posible la creación de las universidades regionales Bluefields Indian Caribbean University y la Universidad de la Regiones Autónomas de la Costa Caribe Nicaragüense (URACCAN, 2004).

A partir de 1995, desde URACCAN se ha promovido la educación para la profesionalización de maestros y maestras como estrategia de autodesarrollo para contrarrestar las condiciones de pobreza y marginación de pueblos indígenas y comunidades étnicas. Esto, en el Caribe Nicaragüense, es un gran esfuerzo paralelo al que hace el gobierno a través del Ministerio de Educación que capacita con limitaciones a docentes de los núcleos educativos. Los Talleres de Evaluación, Planificación y Capacitación Educativa (TEPCES) son eventos que se realizan cada mes, para evaluar la educación del mes anterior y programar la educación del mes que viene, para cada grado de preescolar, primaria y para cada asignatura del currículo de la educación secundaria (MINED, 2008). Los TEPCES no necesariamente son espacios de formación docentes.

En octubre del 2001, la Comisión Técnica del Plan Nacional de Educación (20012015) de Nicaragua incorporó al Sistema Educativo Autonómico Regional (SEAR) un capítulo del Plan, lo que significó un proceso de articulación del SEAR con las políticas educativas a nivel nacional, y a través de los cuales se han realizado procesos 
de transformación curricular en las escuelas normales, en la educación intercultural bilingüe de primaria y en la formación de recursos humanos en diversos niveles, en los cuales URACCAN tiene el importante papel de facilitación y asistencia técnica pedagógica (URACCAN, 2004).

Entre los antecedentes acumulados por URACCAN para mejorar las capacidades y la calidad de docentes de primaria y secundaria están algunas licenciaturas: Ciencias de la Educación con mención en Historia, en Español, o en Inglés; Educación Intercultural Bilingüe, Pedagogía, Ciencias de la Educación con mención en Biología o en Matemática. Por otra parte, existen seis carreras de Profesor de Educación Media con mención en, (i) Ciencias Naturales, (ii) Ciencias Sociales, (iii) Matemática, (iv) Español, (v) Educación Intercultural Bilingüe e (vi) Inglés. Adicionalmente, URACCAN sostiene dos Escuelas de Liderazgo, ocho diplomados en actualización pedagógica en educación intercultural bilingüe para maestros de áreas rurales, de las zonas de Río Coco arriba, Río Coco abajo y en el llano de Tasba Raya en la Costa Caribe Norte (Mato, 2009).

La Región Autónoma de la Costa Caribe Norte cuenta con una cobertura escolar de 132,814 personas en los municipios que comprende el territorio; de las cuales el $17.9 \%$ habita en zonas urbanas y el $82.1 \%$ en zonas rurales. La edad promedio de la población es de 24 años aproximadamente, el 50\% de la población son menores de 19 años y el restante 50\% son mayores de 19 años. La escolaridad promedio de la población es de 2.3 años, o sea aproximadamente 2do. grado de primaria. Un 10\% de la población tiene más de siete grados aprobados, es decir, estudios de al menos primer año de secundaria. De la población de 18 años a menos, el $39.4 \%$ realiza estudios de primaria o secundaria y el 60.6\% no estudia, las razones son problemas económicos, la lejanía de la escuela, el desinterés (MINED, 2008).

La Región Autónoma de la Costa Caribe Sur, cuenta con una cobertura escolar de 103,122 personas en el territorio correspondiente, en donde el $25.5 \%$ habita en zonas urbanas y el $74.5 \%$ en zonas rurales. La edad promedio de la población es de 23 años aproximadamente, el 50\% de la población son menores 17 años y el restante 50\% mayores. La escolaridad promedio de la población es de 2.9 años, es decir aproximadamente tercer grado de primaria; el $50 \%$ de la población tienen al menos dos grados aprobados, el restante $40 \%$ más de dos grados. El 10\% de la población tiene más de ocho grados aprobados, es decir, estudios de al menos segundo año de secundaria. Entre la población menor a 18 años, el $57.4 \%$ realiza estudios de primaria o secundaria y el $42.6 \%$ no estudia; las razones son el desinterés, problemas económicos, el trabajo agrícola (MINED, 2008).

En relación a la Región del Pacifico las desigualdades en educación en la Costa Caribe afectan a la población de manera general, lo que es más evidente en el área rural donde se presenta una escolaridad significativamente inferior, porque las brechas 
entre años de estudios alcanzados y esperados se amplían a medida que aumenta la edad de adolescentes y jóvenes en relación de quienes viven en áreas urbanas. La población adolescente y joven -particularmente del área rural- tiene una considerable privación de capacidades en educación, y por tanto, una alta probabilidad de continuar en pobreza de ingresos (PNUD, 2011). Sin embargo las desigualdades desconciertan en mayor medida a las mujeres de diferentes pueblos indígenas, afro descendientes y comunidades étnicas, por razones indebidas de género, área de residencia, origen étnico, cultura y lengua, debiéndose impulsar transformaciones de cambio (PNUD, 2011).

La educación en la Costa Caribe de Nicaragua cuenta con numerosas barreras, falta de capacidades docentes o de maestros capacitados, de bibliotecas, materiales educativos (textos) y aulas apropiadas-, lo que dificulta la permanencia en el sistema educativo para la terminación de estudios y, aunado a esto, la falta de interés. En relación a esto último, investigaciones realizadas por CIASES (2007) sugieren que la falta de interés está relacionada con la oferta: lo que se enseña no satisface al estudiantado, y para que sea aceptable, debe responder a ciertos criterios de calidad de los contenidos curriculares, las metodologías de enseñanza y los materiales didácticos, entre otros.

La encuesta nacional sobre las percepciones de adolescentes y jóvenes (2009), presentada en el Informe de Desarrollo Humano (2011), brinda información sobre la calidad de la educación; en éste, se expone que se valoró la preparación docente, la forma de enseñanza, los recursos, los contenidos para la educación, la importancia y utilidad de lo que se enseña, y el trato docente, con una apreciación general muy buena entre ocho y diez puntos. Por el contrario, personas jóvenes consultadas en grupo focal Bilwi, en la Región Autónoma de la Costa Caribe Norte de Nicaragua y en el grupo focal Bluefields en la Región Autónoma de la Costa Caribe Sur de Nicaragua, emitieron opiniones más críticas a la educación en cuanto al personal docente, considerando que los principales criterios para evaluar la calidad de la educación son las competencias y el nivel de formación de los docentes y en ambos aspectos su dictamen fue negativo (PNUD, 2011).

La calidad de la educación se evalúa también por los aprendizajes que genera. En los años 2002 y 2006 se aplicaron pruebas realizadas por el MINED para el Estudio Regional Comparativo y Explicativo (SERCE), impulsado por el Laboratorio Latinoamericano de Evaluación de la Calidad de la Educación (LLECE) de UNESCO. Los resultados indican que la mayoría de estudiantes (tercero y sexto grado de primaria) se ubicó en el nivel básico, es decir, tiene muy poco dominio de la materia y un nivel de conocimiento muy inferior al que demanda el currículo, tanto para Español como para Matemáticas. Es minoritario el porcentaje de estudiantes que alcanza un nivel de desempeño competente, es decir, que tiene conocimientos concordantes con lo que demanda el currículo. En este mismo sentido y dirección, se calificó al personal docente en el año 2005. Los resultados mostraron que la escolaridad promedio de los docentes de preescolar, primaria y secundaria correspondía a nueve años (tercer año 
de secundaria), doce años (Primer año de universidad) y catorce años (Tercer año de universidad), respectivamente. En el 2008, el 43\% de los docentes de secundaria no contaban con un título académico en educación; en algunos departamentos, este porcentaje alcanzaba a la gran mayoría de educadores, como en la Región Autónoma del Atlántico Norte (RAAN) de Nicaragua (PNUD, 2011).

La propuesta de competencias que ha de tener el profesor de matemática en la Universidad Comunitaria Intercultural básicamente son:

1. Currículo diversificado, coherente y con distintos modelos de aprendizaje.

2. Trabajar en matemática pensando y comprendiendo.

3. La resolución de problemas como estrategia de enseñanza y aprendizaje.

4. Dominio diverso de la evaluación matemática.

5. Percepción y aceptación del binomio matemática e internet.

6. Conocimiento y dominio de la matemática como ciencia en los campos de:

a. Aritmética y su didáctica.

b. Álgebra básica, lineal, numérica y abstracta

c. Geometría, plana, del espacio, analítica y vectorial

d. Análisis matemático.

e. Probabilidad e inferencia estadística.

f. Investigación educativa: Análisis de problemas actuales en didáctica de las matemáticas.

g. Compromiso con la equidad de género y el respeto a la diversidad cultural, política, religiosa y sexual.

h. En todos los casos se incluye su didáctica

\section{Materiales y métodos}

El presente estudio se enmarca en el paradigma cualitativo porque es una actividad sistemática orientada a la comprensión en profundidad de fenómenos educativos y sociales, a la transformación de prácticas y escenarios socioeducativos, a la toma de decisiones y también hacia el descubrimiento y desarrollo de un cuerpo organizado de conocimiento (Bisquerra, 2012). Desde está perspectiva, Hernández et al. (2010) explican que en el paradigma cualitativo el enfoque o diseño "es el abordaje general que utilizará en el proceso de investigación (p. 492). En este sentido, está investigación tiene un enfoque hermenéutico, porque se interpretaron los significados de textos y acciones en el proceso de formación de profesores de matemática en y para 
la diversidad. Por tales razones, se utilizó como método el análisis de contenido, el cual ayudó a describir los documentos en sus partes esenciales. Para ello, se empleó la técnica de revisión de documento, donde nos centramos en los argumentos, las razones, los propósitos, los significados que los documentos presentan sobre la formación de profesores de matemática. Por consiguiente, se construyó una guía de revisión documental como instrumento, que permitió analizar los libros, artículos científicos, artículos en prensa, leyes, currículo y programas de formación de profesores, políticas y normas institucionales.

\section{Resultados y discusión}

\section{La formación de profesores de matemáticas desde la perspectiva de comunidades de aprendizajes}

La formación de profesores en y para la diversidad es una estrategia de autodesarrollo regional para contrarrestar las condiciones de pobreza y marginación de los pueblos indígenas, afrodescendientes y mestizo de Nicaragua. Entre los antecedentes acumulados por la Universidad Comunitaria Intercultural para mejorar las capacidades y la calidad de profesores de primaria y secundaria están los grados en: Matemáticas y Física-Matemática. Estos profesores son formados desde la metodología de comunidades de aprendizaje, en términos teórico la comunidad de aprendizaje comprende todas las actividades orientadas a la creación, recreación, diseminación e intercambio de conocimientos, saberes, valores y prácticas desde dos vías: la interacción entre conocimientos locales y ancestrales y conocimientos occidentales para el desarrollo de competencias en el mundo del trabajo, el desarrollo de valores y actitudes que preparen a los educandos para enfrentar con éxito los desafíos de la vida (URACCAN, 2014).

A su vez desde un enfoque intercultural, el cual se pronuncia por el desarrollo de un diálogo intercultural como una estrategia para promover espacio de innovación de nuevos conocimientos, ya que confronta elementos de diferentes horizontes y perspectivas culturales, abriendo la posibilidad de impulsar un proceso de complementación y enriquecimiento entre la ciencia moderna y otros saberes (URACCAN, 2013). Así pues, la formación de profesores en y para la diversidad se configura como un proceso de negociabilidad de lo matemática en una comunidad de práctica en la que los aspectos siguientes se manifiestan y se tematizan:

- La interculturalidad: es un proceso de relaciones horizontales donde prevalece el diálogo, a través del cual se propicia el conocimiento mutuo, la comprensión, el respeto, el intercambio y la solidaridad entre los pueblos y las culturas.

- Perspectiva intercultural de género: reconoce y supera las relaciones desiguales entre hombres y mujeres; indígenas, afro-descendientes y mestizos, para lograr construir nuevas relaciones desde sus propias perspectivas, basadas en la 
justicia y equidad, y avanzar hacia el mejoramiento social, económico, político y ambiental en el que su participación responsable y efectiva en el ejercicio de sus derechos y deberes, en la toma de decisiones sobre políticas públicas, fortalece la autonomía regional y por ende el ejercicio efectivo de la ciudadanía intercultural.

- Autonomía regional: respeto y promoción de un sistema de administración, autogobierno y autodeterminación, a nivel comunal, territorial, municipal y regional, que conlleva al Buen Vivir de los pueblos culturalmente diferenciados en unidad en la diversidad.

- Buen vivir y desarrollo con identidad: comprende el desarrollo de procesos para lograr la complementariedad, armonía y equilibrio entre la Madre Tierra y las sociedades multiculturales.

- Diálogo de saberes: acciones que llevan a crear, recrear, compartir, diseminar e intercambiar conocimiento, saberes y prácticas desde la interacción entre el conocimiento propio fundamentado en la espiritualidad y el conocimiento occidental.

Resulta evidente que el profesor que atiende poblaciones en contexto de diversidad necesita estar preparado para impartir contenidos contemplados en el currículo nacional y formar adecuadamente a sus estudiantes. Por ello, en los currículos de formación de profesores en y para la diversidad se introducido el estudio de las matemáticas en ambientes interculturales y pluritecnológicos para que los profesores en proceso de formación estén capacitados para asumir las responsabilidades que esta nueva sociedad les encomienda.

\section{La formación de profesores de matemáticas desde la creación y recreación de conocimientos, saberes y prácticas}

En la Universidad Comunitaria Intercultural la investigación se define como un proceso científico y participativo que genera conocimientos, capacidades e innovaciones; además sistematiza y revaloriza las prácticas históricas y saberes locales de los pueblos indígenas, afrodescendientes y comunidades mestiza en la búsqueda del desarrollo pleno y sostenible desde la perspectiva autonómica (URACCAN, 2014). Su misión es contribuir al desarrollo y fortalecimiento de la autonomía de la Costa Caribe nicaragüense a través de la generación, sistematización, publicación, difusión de los conocimientos científicos, así como la revitalización de las prácticas tradicionales, saberes locales de los pueblos indígenas, afrodescendientes y comunidades mestiza, mediante la participación activa de la universidad, investigadores asociados y actores locales, como estrategia para el desarrollo de capacidades emprendedores y de innovación.

En su visión se propone ser referente de un proceso que genera cambios, constituye propuesta y sistematiza experiencias que contribuyan al conocimiento científico 
y al fortalecimiento de la identidad en el marco de la autonomía de la costa caribe nicaragüense, a través de la promoción de iniciativas innovadoras y emprendedores de autogestión comunitaria y regional (URACCAN, 2005). Permite llevar a las aulas de clases el conocimiento ancestral, que por tanto tiempo ha estado en los pueblos indígenas y que sólo ha servido para usarlo como referencia de estudios sin darles el crédito debido. Los procesos investigativos deben respetar los derechos de propiedad intelectual colectiva de los pueblos indígenas y afrodescendientes sobre sus conocimientos. Debe plantearse un convenio de ética cuando se trabaje en las comunidades, que permita a las parte involucradas en esta experiencia aprovechar al máximo los saberes, pero al mismo tiempo que haya satisfacción en lo aportado y acuerdo en lo divulgado (URACCAN, 2012).

Lo anterior permite fundamentar las premisas de creación y recreación de conocimientos saberes y prácticas; innovación; innovación comunitaria; emprendimiento URACCAN (2014):

1. Creación y recreación de conocimiento saberes y prácticas: En URACCAN la creación y recreación de conocimiento, saberes y prácticas se define como un proceso participativo que genera nuevos conocimientos, capacidades e innovaciones. Sistematiza y revaloriza las prácticas históricas y saberes locales de los pueblos indígenas, afrodescendientes, mestizas y comunidades étnicas en la búsqueda del buen vivir.

2. Innovación: Se entiende que la innovación es el proceso de crear o recrear un nuevo o significativamente mejorado producto, proceso o método.

3. Innovación comunitaria: representa una plataforma eficaz para la construcción de conocimientos, parte de la base que existen sistemas de conocimientos locales autóctonos, así como prácticas que han hecho posible la supervivencia de los pueblos y comunidades así como la defensa de sus recursos naturales.

4. Emprendimiento: Es la manera de pensar, sentir y actuar, en la búsqueda de iniciar, crear o formar un proyecto a través de la identificación de ideas y oportunidades de negocios. Es crear nuevas ideas y formas de hacer las cosas con iniciativas y decisión ante las distintas circunstancias, que permitan la creación de empresas, generación de autoempleo, crecimiento de empresas ya existentes y formación de redes.

En este contexto, el estado del arte de las investigaciones que se han publicado en revista científicas y libros, desde las perspectivas de las matemáticas son: 


\section{- Investigaciones de las perspectiva sociocultural de las matemáticas}

Winsing y Torres (2010) realizan una investigación sobre los elementos lingüísticos que obstaculizan el aprendizaje de los nombres de los números naturales en Miskitu, está investigación destaca lo siguiente: i) existen cambios en los nombres de los números naturales; ii) evidentes dificultades lingüísticas en los nombres de los números naturales del miskitu; iii) los nombres de los números naturales del sistema de numeración miskitu en uso actual, tiene indicio de sub-base quinaria y base vigesimal. En este mismo camino, Soza y Dávila (2012) hacen un estudio sobre "Etnomatemática en indígenas Ulwas, comunidades de Karawala, Región Autónoma Atlántico Sur, Nicaragua" y ponen de manifiesto que el sistema numérico del conteo con los dedos; este conteo, los ancestros ulwas lo hacían con los dedos de las manos y de los pies, en el sistema de medición se identificaron como unidades de medida la vara, la jícara, los nudos en bejuco. También el empleo de conocimiento geométricos en la construcción de viviendas tradicionales, nasas y elaboración del metate. Los aportes de esta investigación contribuyen en la revitalización cultural de los pueblos indígenas ulwas y a la gestión curricular en el sub sistema educativo autónomo regional (SEAR), los cuales deben ser adoptados en la transformación curricular de los programas de la educación intercultural bilingüe.

Así mismo, Gutiérrez y Rojas (2013) ponen en claro las "expresiones del cálculo en la cultura Miskitu de Sandy Bay Sirpi, Región Autónoma de la Costa Caribe Sur", los autores realizan una clasificación de seis actividades etnomatemáticas fundamentales y universales de grupos culturales, tales como contar, localizar, medir, diseñar, jugar y explicar. En el conteo, el pueblo miskitu tiene desde tiempos antiguos su propia numeración en idioma miskitu. De igual forma, realizan mediciones tanto de longitud como de masa y peso, utilizando, las partes del cuerpo (manos, pies y dedos), materiales como guacales y madera. En cuanto a las medidas del tiempo: el sol, así como el ciclo lunar y el calendario miskitu, son las principales estrategias de localización. Por otro lado, en la construcción de viviendas, cayucos y otros se utilizan diferentes figuras geométricas.

\section{- Investigaciones desde la perspectivas de diseños didácticos}

Gutiérrez y López, (2010) realizan un diseño didáctico sobre la "enseñanza de la geometría en segundo año de educación secundaria bajo el enfoque de competencias", el estudio se centró en: (a) las metodologías de la enseñanza de los profesores de matemáticas en el desarrollo de los contenidos geometría plana de segundo año, implementando el enfoque por competencias y (b) la manera en que estas contribuyen al aprendizaje que adquieren los estudiantes de geometría. En Gómez, Medina y López (2010) proponen una metodología basada en competencias para la enseñanza de gráficas y análisis de funciones trigonométricas. Entre los resultados más relevante de esta investigación está en que todos los estudiantes a los cuales se le aplicó el examen 
experimental no pueden graficar funciones trigonométricas; además, se les hace muy difícil analizar la solución de ejercicios por lo que no recuerdan las definiciones de las funciones trigonométricas. La investigación concluye que se hace necesario de la utilización de nuevas metodologías de enseñanza para impartir este tema.

También, Flores y Salinas (2013) describen las metodologías que utilizan profesores de matemáticas, en la enseñanza-aprendizaje de la Derivada a estudiantes de Administración de Empresas, y cómo estas metodologías educativas influyen en el rendimiento de la asignatura "Matemática Financiera". Fue un estudio de caso en el que participaron dos profesores de matemática; los datos fueron obtenidos a través de un cuestionario y entrevista abierta; además, una encuesta que se le suministró al alumnado en las que se les solicitó información relacionada a los procesos metodológicos en el aula. El análisis se desarrolló en función de las redes sistémicas y los resultados muestran que existen diferencias significativas a la hora de abordar el contenido, los resultados comparativos favorecen a los estudiantes a quienes se les facilitó clases en la enseña de la Derivada con pertinencia al contexto social y en cuanto al profesor que aborda la Derivada desde el enfoque tradicional, se evidencian calificaciones bajas por parte del estudiantado.

\section{- Investigaciones desde la perspectiva del reconocimiento de la diversidad en la formación de profesores en y para la diversidad}

En CAM (2013) se plantean orientaciones específicas para la incorporación de tecnología en procesos de formación de profesores de matemática en contexto de diversidad para el diseño de secuencias didácticas. Dichas orientaciones, se dirigen a los formadores de profesores, estudiantes para profesores y profesores en ejercicios del área de matemática, como usuarios naturales para la propuesta. Considerando específicamente que las orientaciones constituyen un potencial para el desarrollo y la formación profesional del profesor de matemáticas y presentan el uso de tecnologías como una mediación para una educación matemática en y para la diversidad. Igualmente, CAM (2014) sitúa "referentes curriculares con incorporación tecnológica para la formación del profesorado de matemáticas en y para la diversidad", la investigación presenta el resultado de muchas discusiones, reflexiones, análisis y trabajo conjunto que dieron origen a consenso fundamentados, como sucede con el reconocimiento de la necesidad de incorporar en la elaboración de los referentes curriculares seis aspectos fundamentales:

(i) Las múltiples experiencias con la matemática y su didáctica.

(ii) Las múltiples representaciones de lo matemático y de su didáctica.

(iii) Los múltiples tipos de interacción en los ambientes de aprendizaje.

(iv) Las poblaciones en vulnerabilidad educativa por sus condiciones sensoriales, étnicas y económicas. 
(v) La presencia de las Tecnologías de la Información y Comunicación.

(vi) Una metodología de interacción entre diversos y de desarrollo co-operativo, definida por las comunidades de práctica.

Por su parte, Flores y Auzmendi (2015a) ponen de manifiesto que la integración de las tecnologías de la información y comunicación en procesos de formación de profesores de matemáticas en y para la diversidad, debe ser articulada desde los componentes de integración tecnológicos en los ambientes mediados por TIC; los diseños didácticos; la gestión didáctica y la evaluación educativa. Así mismo, Flores y Auzmendi (2015b) confirman que los ambientes interculturales y pluritecnológicos desarrollan el aprendizaje de las matemáticas en y para la diversidad, son un hacer natural en el aprendizaje de las matemáticas que es objeto de su enseñanza son los ambientes interculturales y pluritecnológicos. Ambientes que no pueden limitarse a distribuir contenidos sino que le supone proponer un conjunto de actividades y de tareas que orienten las experiencias en las que participarán los estudiantes, teniendo como referencia la diversidad y la incorporación tecnológica, así, los profesores, han de asumir como una de sus tareas, que delimitan y caracterizan su actuación, el seleccionar, organizar, y planificar las experiencias de aprendizaje necesarias para que un estudiante aprenda en ambientas diversos.

\section{- Investigaciones desde la perspectiva de las actitudes hacia las matemáticas y estadísticas}

En Flores y Auzmendi (2015c) se realiza un análisis estructural de una escala de actitud hacia las matemáticas para poblaciones en contexto diversidad a partir de una muestra de 182 estudiantes universitarios de especialidad de educación (Biología, Inglés, Español, Historia, Pedagogía) el estudio consistió en analizar las propiedades psicométricas y el análisis en las puntuaciones medias de la escala de actitud hacia la matemática. Concluyendo que existen diferencias estadísticamente significativos $\mathrm{y}$, en todos los casos, los estudiantes de la especialidad en Inglés registran puntuaciones más altas con respectos a las demás especialidades y la escala tiene un modelo adecuado, lo que permitiría su uso en contextos diversos. Así mismo, Flores (2015a), hace un estudio sobre las actitudes hacia la estadística en procesos de formación de profesores en y para la diversidad con una muestra de 276 estudiantes universitarios de especializaciones en ciencias de la educación (Cultura Física, Español, Inglés, Educación Intercultural, Pedagogía, Biología) los resultados sitúan a las componentes afectivo, cognitivo y dificultad estrechamente correlacionadas, sin embargo, el componente valor se diferencia del resto; también se muestran las existencias de diferencias estadísticamente significativas y, en todos los casos, los estudiantes de la especialidad en Cultura Física, Inglés y Educación Intercultural registran puntuaciones más altas con respectos a las demás especialidades. Se concluye que el aprendizaje estadístico en procesos de formación de profesores en y para la diversidad mejora sus actitudes. 


\section{- Investigaciones desde la perspectiva de la comprensión de los conceptos matemáticos}

En Gaitán, Lacayo y Flores (2014) se estudia la comprensión del aprendizaje de la parábola en undécima grado aplicando el modelo de Van Hiele, la investigación describe las dificultades de comprensión en el aprendizaje de la parábola en estudiantes de undécimo grado y algunos aspectos de concepción del profesor de matemática desde el punto de vista de la evaluación educativa. Los resultados muestran que las principales dificultades de la comprensión en el aprendizaje del contenido de la parábola, están vinculadas con las representaciones propias de los estudiantes con respecto al contenido, además de dificultades de comunicación docente-discente durante el proceso de instrucción Matemática con los cuales podemos caracterizar que los niveles del razonamiento de los estudiantes están vinculados a las diversas experiencias en el medio, permitiendo entrelazar imágenes como representaciones cognitivas o conceptuales a modelos del conocimiento matemático.

Así mismo, Flores (2015b) analiza los problemas del álgebra a través de la adaptación y validación de un cuestionario para su medida, en dicha investigación participaron 258 estudiantes de Ciencias Administrativas e Informática pertenecientes de comunidades indígenas, afrodescendientes y mestizo de Nicaragua. Los resultandos brindan, la problemática alrededor de los contenidos de álgebra, y se concluye que el cuestionario es un instrumento que forma parte de un engranaje global frente al cual se puede aplicar en contextos diversos.

\section{La formación de profesores de matemática como estrategia de acompañamiento e incidencia social y comunitaria}

El acompañamiento e incidencia social y comunitaria se define como un proceso de diálogo de saberes implementado por la universidad con las comunidades y actores sociales; encaminados al ordenamiento de desarrollo regional y fortalecimiento de los sistemas autonómicos y la promoción del patrimonio cultural desde un enfoque de equidad de género, para la construcción colectiva del Buen Vivir de los pueblos, el respeto, equilibrio y armonía con la Madre Tierra a nivel nacional e internacional (URACCAN, 2014). Desde estas acciones la formación del profesor de matemática se centra en un proceso de investigación educativa, especialmente relacionado a los procesos de enseñanza-aprendizaje de las matemáticas en contexto multiculturales tendientes a consolidar un sistema educativo acorde a las necesidades y características de los pueblos indígenas, afrodescendientes, mestizo y comunidades étnicas de la región y el país.

El acompañamiento e incidencia social comunitaria enriquece la formación integral del estudiante para profesor de matemáticas ya que lo vincula con la realidad que lo rodea y lo involucra en la atención de problemas y necesidades de su entorno social. La estrategia de acompañamiento e incidencia social y comunitaria en la formación 
de profesores de matemáticas es retomada desde la investigación acción participativa que trata de promover las habilidades, destrezas, actitudes y valores de una práctica investigativa en el transcurso de la formación del futuro profesor de matemática. Además de fomentar al estudiantado la revitalización de procesos matemáticos a través de un análisis de las potencialidades, tradiciones, costumbres, necesidades e intereses de los comunidades indígenas, afrodescendientes y mestiza de la Costa Caribe Nicaragüense. En este contexto, el currículo de formación de profesores de matemática se enlaza con los conglomerados siguientes:

- Metodología de la Investigación Social. La metodología de la investigación social pretende proporcionar a los estudiantes las nociones básicas de la metodología de la indagación científica en su aplicación a las ciencias de la educación. Familiarizar a los estudiantes con diferentes diseños de investigación. Desarrollar la capacidad de utilizar los principales conceptos, métodos, técnicas e instrumentos utilizados en el proceso de investigación.

- Investigación y Práctica Educativa. La investigación y práctica educativa tiene como objetivo introducir a los estudiantes en el campo de la investigación educativa, con énfasis en la realización de aproximaciones a las diversas realidades educativas. Se aborda la utilización de técnicas para el diagnóstico cualitativo y cuantitativo de necesidades dentro de instituciones educativas. Proporciona a los estudiantes las herramientas metodológicas necesarias para indagar sobre distintos problemas pedagógicos. Además, entrega técnicas de análisis, presentación de datos y aplicación de programas computacionales, y presentación de informes a través de los cuales darán cuenta de sus hallazgos. El curso busca desarrollar en los estudiantes la capacidad y estilo de trabajo, orientando sus futuras prácticas pedagógicas, de manera que tengan herramientas eficaces para enfrentar los diversos problemas del trabajo pedagógico diario en forma crítica, científica y profesional.

- Diagnóstico Comunitario (Investigación Acción I). Esta práctica ofrece oportunidades para acercarse a la realidad desde la perspectiva científica para identificar, describir y analizar las características, problemas, potencialidades, tradiciones, costumbres, necesidades e intereses de la comunidad; en esta carrera especialmente en relación a la educación y a las matemáticas. Está orientada también a aplicar los aprendizajes adquiridos o desarrollados en las diferentes asignaturas del primer año de formación. El tiempo lectivo del curso correspondiente es un espacio para compartir la experiencia de la práctica y retroalimentarla técnicamente.

- Diversidad en el Aula (Práctica de Investigación Acción II). Concebida como una práctica de observación y reflexión, pretende acercar al estudiante con el fenómeno de la diversidad sociocultural en contextos educativos y la manera 
en que se aborda ésta. Se ofrecen instrumentos y experiencias para captar las diferencias socioculturales de las y los educandos, las convergencias, las ventajas, los problemas y los desafíos de la confluencia multicultural en los centros educativos. También se analiza la actitud del educador frente a la diversidad en el aula y se discuten pautas positivas de actuación. El tiempo lectivo del curso correspondiente es un espacio para compartir la experiencia de la práctica y retroalimentarla técnicamente.

- Investigación Educativa (Práctica de Investigación III). Esta actividad curricular es una experiencia concreta de reflexión al enfrentarse a la detección y análisis de problemas educativos a nivel de aula, Constituye la continuidad de las prácticas anteriores en la cual el estudiante selecciona, investiga y analiza una necesidad específica y relevante, detectada en una realidad particular. Se orienta al estudiante para que trabaje alrededor de su especialidad, el aprendizaje de matemática; especialmente porque se espera que el problema que identifique lo continúe trabajando en otros cursos para ir constituyendo su proyecto de graduación. El tiempo lectivo del curso correspondiente es un espacio para compartir la experiencia de la práctica y retroalimentarla técnicamente.

- Elaboración de Proyectos Educativos (Práctica de Investigación IV). Esta práctica está orientada a la formulación de un proyecto educativo innovador de carácter pedagógico- en respuesta a una necesidad o problema debidamente identificado en la práctica de Investigación Educativa. El estudiante se enfrenta con un problema concreto, ante el cual debe proponer alternativas viables y efectivas para su solución. Es una oportunidad de aplicar los conocimientos adquiridos en todos los cursos. Se orienta al estudiante para que trabaje alrededor de su especialidad, el aprendizaje de la matemática. Los productos de este curso podrá mejorarlos para presentar su proyecto de graduación. El tiempo lectivo del curso correspondiente es un espacio para compartir la experiencia de la práctica y retroalimentarla técnicamente.

\section{La formación de profesores de matemáticas como proceso de gestión y comunicación intercultural para el desarrollo institucional}

En la Universidad Comunitaria Intercultural la función gestión y comunicación intercultural para el desarrollo institucional consiste en el proceso de gerencia pertinente y de calidad a través de la construcción concertada de planes institucionales donde se implemente, monitorea, acompaña y evalúa a todos los niveles, tareas y procedimientos que garantizan el desarrollo efectivo de las funciones básicas de la institución. La gerencia, garantiza la implementación de las políticas, normativas y planes institucionales asegurando los recursos técnicos y financieros necesarios. En este proceso la comunicación intercultural es el instrumento base para la articulación y gerencia efectiva de las distintas instancias, haciendo posible el cumplimiento de la misión, visión y objetivos institucionales (URACCAN, 2014). 
La gestión y comunicación intercultural son actividades que posibilitan instaurar procesos de negociación de significados en aulas de didáctica de las matemáticas con comunidades de profesores en y para la diversidad. Por ello, la Universidad Comunitaria Intercultural profesionaliza a sus profesores de matemáticas bajo principios de autonomía, interculturalidad, solidaridad, pertinencia, calidad, equidad, -especialmente género- valores éticos y cívicos y cultural regional y nacional, para alcanzar el desarrollo sostenible en lo económico, social, político y cultural. Todo anudando a un objetivo que valora la educación con función social "educar a costeños y costeñas comprometidos y comprometidas con el desarrollo humano sostenible de su comunidad y región y con la práctica permanente de la interculturalidad con una sólida preparación, técnica y humanista orgullosos de su identidad étnica, cultural y lingüistica y que contribuyan al proyecto de unidad nacional en la diversidad" (URACCAN, 2010, p.14).

Y específicamente recomienda que la gestión y comunicación intercultural en la formación de profesores de matemáticas donde se involucran poblaciones indígenas, afrodescendientes y mestiza debe considerar:

- Desarrollar un sentido positivo de sí mismo y expresar sus sentimientos, actuando con iniciativa, autonomía y percibiendo sus logros al realizar actividades individuales y de colaboración;

- Asumir roles distintos en el juego y en otras actividades para trabajar en colaboración, apoyarse entre compañeros y compañeras, resolver conflictos a través del diálogo y reconocer y respetar las reglas de convivencia en el aula, en la universidad y fuera de ella;

- Reconocer los rasgos culturales distintos (lenguas, tradiciones, formas de ser y de vivir) de las personas y grupos, compartiendo experiencias de su vida familiar y fuentes de información (otras personas, medios de comunicación masiva a su alcance: impreso, electrónicos);

- Expresarse, dialogar y conversar en su lengua materna, mejorar su capacidad de escucha, ampliar su vocabulario y enriquecer su lenguaje oral en diversas situaciones y contexto comunicativos.

Además, avisa que el conocer términos que se utilizan en diferentes grupos y reconocer su significado (palabras, expresiones, canciones o textos); aprender a rechazar cualquier discriminación por condición u origen social, género o etnia, nacionalidad u orientación cultural, sexual, religiosa o contexto de hábitat, condición física, intelectual o lingüística; ser capaces de resolver problemas de manera creativa mediante situaciones de juego que impliquen la reflexión, la explicación y la búsqueda de soluciones a través de estrategias o procedimientos propios, y su comparación con los utilizados por otros; interesarse en la observación de fenómenos naturales, participando en 
situaciones de experimentación que abran oportunidades para preguntar, predecir, comparar, registrar, elaborar explicaciones e intercambiar opiniones sobre procesos de transformación del mundo natural y social inmediato, adquiriendo actitudes favorables hacia el cuidado y la preservación del medio ambiente, que recuperen los saberes y experiencias de los pueblos indígenas y afro descendientes.

\section{La formación de profesores de matemática como proceso de cooperación, solidaridad y complementariedad nacional e internacional}

Los proceso de cooperación, solidaridad y complementariedad es una plataforma amplia de solidaridad en permanente construcción a partir de la realización del paradigma de universidad comunitaria intercultural y del Buen Vivir, sustentada por una constante búsqueda de una relación armónica del conocimiento científico-técnico útil con el conocimiento y los saberes ancestrales de las comunidades indígenas, afrodescendientes y mestiza de la Costa Caribe de Nicaragua (URACCAN, 2013b). En este diálogo epistemológico intercultural, desde la formación de profesores de matemáticas se prioriza "la formación de profesionales en Ciencias de la Educación con Mención en Matemáticas con sólidas bases científicas, tecnológicas y humanísticas que faciliten servicios educativos de calidad acorde a las exigencias del país y particularmente las de la Costa Caribe de Nicaragua, especialmente en lo relativo al respeto y desarrollo de la pluriculturalidad, el desarrollo sostenible y el fortalecimiento del proceso de autonomía" (URACCAN, 2010, p. 23)

Desde esta perspectiva, la formación de profesores de matemáticas como proceso de cooperación, solidaridad y complementariedad se articula con el modelo pedagógico de la Universidad Comunitaria Intercultural a partir de los fundamentos teóricos que ofrecen las pedagogías iluminadas por el enfoque histórico cultural. Se considera valioso orientar la formación hacia aspectos educativos, tales como:

1. Formación multidimensional que considera a los sujetos como una unidad múltiple que integra lo biopsicosocial, individualidad e historia, en relación íntima con el carácter, temperamento, corporeidad física y desarrollo corporal.

2. Una visión dialéctica de Sociedad - Educación que promueve la transformación de la sociedad, desde las bases estructurales y súper estructurales.

3. Capacidad para comprender y afianzar la articulación entre la educación y la esfera macro-social.

4. Responsabilidad con el medio social, compromiso y solidaridad con las particularidades del contexto en que se desarrolla el aprendizaje. 
Estos aspectos educativos se configuran en asignaturas como las siguientes:

- Ciudadanía Intercultural: consiste en la capacidad de los pueblos indígenas, afro descendientes y mestizo de ejercer sus derechos individuales y colectivos, teniendo para ello acceso con equidad social a la educación, salud, bienestar material, trabajo y participación, en base a su propia manera de ver el mundo.

- Educación, género y sentido crítico: reside en la identificación de las situaciones de exclusión en que viven las mujeres en las sociedades actuales y promueve una revisión crítica de cómo la escuela reproduce estereotipos y roles tradicionales al respecto. Posteriormente, propone criterios y lineamientos para la formación de la conciencia de género tanto en hombres como en mujeres, de cara al establecimiento de relaciones de equidad, justicia y cooperación.

- Educación y sociedad: se centra en las relaciones de la educación y la sociedad. La familia y la escuela, la comunidad y la escuela. Pilares fundamentales de la Educación. Pertinencia, flexibilidad, relevancia e integralidad de la educación.

- Educación Intercultural: se fundamenta en explorar los tipos de relaciones que históricamente se han establecido entre pueblos de culturas diferentes (exterminio, dominación, así mismo, fusión cultural, multiculturalismo, entre otros.) y plantear los conceptos básicos para comprender el modelo de relaciones interculturales. Desarrolla, como un componente fundamental de la convivencia intercultural. Define los conceptos de lengua primera, lengua materna, lengua extranjera y segunda lengua. Presenta el reto de educar para convivir de forma positiva en la diversidad cultural. Aborda el desafío de aprovechar la diversidad cultural para fomentar el intercambio positivo -aprender del otro-, sin lastimar las identidades y culturas particulares.

\section{Conclusiones}

Los procesos de formación de profesores de matemática se relaciona fundamentalmente con cambios en los entornos sociales, en los estudiantes y en la educación, y sigue la dinámica de la cinco funciones sustantivas de la Universidad Comunitaria Intercultural: Comunidades de aprendizaje; creación y recreación de conocimientos, saberes y prácticas; estrategia de acompañamiento e incidencia social y comunitaria; gestión y comunicación intercultural para el desarrollo institucional; cooperación, solidaridad y complementariedad nacional e internacional. Dichas funciones son sustantivas para la formación de profesores de matemáticas. Por lo tanto, en la formación profesor de matemáticas se debe reconocer:

- El papel que juega la comunidad en la universidad, los sabios, sabias, ancianos, ancianas y autoridades tradicionales y no tradicionales en los procesos educativos; 
- La búsqueda de nuevos paradigma en los enfoques pedagógicos y metodológicos para le generación de conocimientos para el Buen Vivir de los pueblos;

- La espiritualidad y el pensamiento mágico religioso;

- La interculturalidad, la educación intercultural y la educación multilingüe;

- La igualdad de género;

- El papel central de la investigación y la innovación;

- La articulación entre la teoría y la práctica;

- Educación para todos y todas a lo largo de toda la vida.

La formación de profesores de matemáticas no pueden desvincularse de las otras disecciones didácticas: principios del acto comunicativo, manifestaciones de la comunidad de práctica, formación multidimensional, visión dialéctica, capacidad para comprender, responsabilidad social. Es decir, formar profesores de Matemática desde la perspectiva de la Universidad Comunitaria Intercultural constituye un hacer natural en el aprendizaje de las matemáticas para las poblaciones indígenas, afrodescendientes y mestiza de la Costa Caribe de Nicaragua. Formación que no se limita a distribuir contenidos sino que le supone un conjunto de actividades, tareas y prácticas compartidas que orientan las experiencias en las que participarán los futuros profesores de matemáticas.

En general, en los procesos de formación de profesores de Matemática se reconoce y se asume que los pueblos indígenas son portadores milenarios de conocimientos y saberes, que sus culturas enriquecen la cultural global. Son de gran valía los valores éticos, humanos y espirituales que los pueblos indígenas trasmiten. La cultura y la relación que los pueblos indígenas asumen con el medio ambiente -su cosmovisión y cosmogonía-, son fuente inagotable y constituyen una energía revitalizadora y sustentable de saberes y conocimientos, que pueden ser fuentes científicos en el ámbito teórico y práctico para el autodesarrollo en el marco de una nueva relación horizontal con el mundo no-indígena. 


\section{Lista de referencia}

Bisquerra, R. (2012). Metodología de la Investigación Educativa. Madrid: La Muralla.

CAM (2013). Orientaciones especificas para la incorporación de tecnología en procesos de formación de profesores de Ciencias Naturales, Lenguaje y Comunicación y Matemáticas en contexto de diversidad para el diseño de secuencias de enseñanzaaprendizaje. Valparaíso: Ediciones Universitarias de Valparaíso.

CAM (2014). Referentes curriculares con incorporación de tecnologías para la formación de profesores de matemáticas en y para la diversidad. México: Ediciones de la Universidad Pedagógica de México.

Flores, W., O., \& Salinas, M. (2013). Metodologías en la enseñanza de la derivada en la URACCAN-Nueva Guinea. Revista Ciencia e Interculturalidad, Vol. 12, № 1, 39-49. http://revistas.uraccan.edu.ni/index.php/Interculturalidad/article/view/381

Flores, W., O. (2015a). Actitudes hacia la estadística en procesos de formación de profesores en y para la diversidad. (En Prensa).

Flores, W., O. (2015b). Los problemas del álgebra: adaptación y validación de un cuestionario para su medida. Revista Horizontes Pedagógicos. (En Prensa).

Flores, W., O., \& Auzmendi, E. (2015a). Integración de las tecnologías de la información y comunicación en la formación de profesores de matemáticas en y para la diversidad. En C. Varela, A. Miñán \& L. Bengochea. (Eds.) Formación virtual inclusiva y de calidad para el siglo XXI (318-326). Granada: Universidad de Granada.

Flores, W., O., \& Auzmendi, E. (2015b). Ambientes Interculturales y Pluritecnológicos desarrollan el aprendizaje de las matemáticas en y para la diversidad. En C. Varela, A. Miñán \& L. Bengochea. (Eds.) Formación virtual inclusiva y de calidad para el siglo XXI (318-326). Granada: Universidad de Granada.

Flores, W., O., \& Auzmendi, E. (2015c). Análisis de la estructural factorial de una escala de actitud hacia las matemáticas. Revista Aula Encuentro. (En Prensa).

Gaitán, A., M., Lacayo, M., A., \& Flores, W., O. (2014). Comprensión del aprendizaje de la parábola en undécimo grado aplicando el modelo de Van Hiele. Revista Ciencia e Interculturalidad, 21-33. 
Gaceta (2007). La constitución política de Nicaragua. En corte suprema de justicia (Eds.) La constitución políticas y sus reformas, 1-28. Managua: Biblioteca jurídica. http://www.poderjudicial.gob.ni

Gómez, B., Medina, M., \& López, E. (2010). Propuesta Metodológica basada en competencias para la enseñanza de gráficas y análisis de funciones trigonométricas. Revista Ciencia e Intercultural, Vol. 7, $N^{\circ}$ 2, 125-133. http://revistas.uraccan. edu.ni/index.php/Interculturalidad/article/view/45

Gutiérrez, S., \& López, E. (2010). Enseñanza de la geometría en segundo año de educación secundaria bajo el enfoque de competencia: una propuesta metodológica para la enseñanza de la geometría. Revista Ciencia e Interculturalidad, Vol. 6, $\mathrm{N}^{\circ} 1$ 1, 110-119. http://revistas.uraccan.edu.ni/index.php/Interculturalidad/article/view/71

Gutiérrez, S., \& Rojas, N. (2013). Expresiones del cálculo en la cultura Miskitu de Sandy Bay Sirpi, Región Autónoma del Atlántico Sur. Revista Ciencia e Interculturalidad, Vol. 12, $\mathrm{N}^{\circ}$ 1, 8-20. http://revistas.uraccan.edu.ni/index.php/Interculturalidad/ article/view/379/322

Hernández, R., Fernández, C., \& Baptista, M. (2010). Metodología de la Investigación. México: McGraw-Hill.

Mato, D. (2009). Instituciones Interculturales de Educación Superior en América Latina. Procesos de Construcción. Logros, Innovaciones y Desafíos. Caracas: IESALC-UNESCO.

MINED (2008). Informe de Estadística y Censo del Ministerio de Educación de Nicaragua. Managua: División de Estadística.

Sánchez, A. (2009). Un recuento del proceso autonómico en Nicaragua. Desacatos, 31 (3), 173-179.

PNUD (2011). Informe Nacional sobre Desarrollo Humano 2011: Las juventudes construyendo Nicaragua. Managua: La prensa.

URACCAN (2004). Formación para la ciudadanía intercultural. Bluefields: IPILC-URACCAN.

URACCAN (2005). Política Institucional de Investigación. Managua: URACCAN.

URACCAN (2010). Currículo de la Licenciatura en Ciencias de la Educación con Mención en Matemáticas. Nueva Guinea: URACCAN. 
URACCAN (2012). Régimen Académico Institucional. Nueva Guinea: URACCAN.

URACCAN (2013a). Proyecto Educativo Institucional. Nicaragua: URACCAN.

URACCAN (2013b). Política de gestión intercultural de cooperación externa de URACCAN con perspectiva intercultural de género. Bilwi: URACCAN.

URACCAN (2014). Plan Estratégico Institucional 2015-2019 de URACCAN. Nicaragua: URACCAN.

Winsing, V., \& Torres, A. (2010). Elementos lingüísticos que obstaculizan el aprendizaje de los nombres de los números naturales en Miskito. Revista Ciencias e Interculturalidad, Vol. 6, $\mathrm{N}^{\circ} 1$, 45-57. http://revistas.uraccan.edu.ni/index.php/ Interculturalidad/article/view/61/43 